\title{
The Topography and Location of Nanoparticles in Supported Catalysts using Back Scattered Electrons and HAADF-STEM in a FESEM
}

\author{
Pratibha L. Gai ${ }^{* * *}$ and D.L. Smith ${ }^{*}$ \\ * DuPont, Central Research and Development, Experimental Station, Wilmington, DE 19880- \\ 0356; ${ }^{* *}$ Department of Materials Science, University of Delaware, Newark, DE.
}

Methods for determining the size, chemical composition and morphology of nanocatalysts dispersed on high surface area supports are of key importance in heterogeneous catalysis. The principles underpinning chemical microanalysis of bulk samples in the SEM using low beam voltages and energy dispersive X-ray spectroscopy have been reported [1]. Recently efforts have been focused on ultrasensitive methods including Z-contrast imaging using high angle annular dark field (HAADF) detector in a high resolution scanning-TEM (HRSTEM) [2]. HAADFHRSTEM method exploits the fact that high angle scattering intensity of electrons from thin samples follows the $Z^{2}$ dependence of Rutherford's law where $Z$ is the atomic number of the element. High angle electrons recorded using HAADF detector offer essentially an incoherent signal and images are monotonically dependent on the atomic number of the sample. Studies using both back scattered electrons (BSE) which also utilize electrons scattered at high angles and HAADF-STEM imaging in a scanning EM to obtain spatial distribution and morphology of nanocatalysts have been reported by Gai et al $[3,4]$. In this paper, we present comparisons of HAADF-STEM and BSE of a number of supported catalysts at the same electron energies recorded in a field emission scanning electron microscope (FESEM) equipped with a BSE detector and a HAADF-STEM detector in the same instrument.

A variety of industrial catalytic processes employ nanometallic particle systems on supports. These nanocatalysts contain transition metals or noble metals or alloys or bimetallic systems. Commonly used supports are carbon and ceramic oxides including silica, alumina and zeolites. The supports are low Z and high surface area solids. FIG. 1(a) and (b) show BSE and HAADFSTEM images, respectively, of the same area of shadowed gold on carbon, recorded at $30 \mathrm{kV}$ in the FESEM. FIG. 2(a) and (b) show commercially important Pd nanocatalysts on carbon in BSE and HAADF-STEM, respectively, at $30 \mathrm{kV}$ in the FESEM. The metallic nanoparticles are on uneven, thicker supports. The data show that similar high resolution images are obtained in either BSE or forward-scattered (HAADF) mode. The studies show considerable potential of the methods in the SEM in the analysis of catalysts on non-uniform supports and similar nanostructured systems. The images also illustrate that BSE and HAADF in an SEM may be more advantageous compared to the conventional BF-TEM and BF-STEM which generally operate at $100 \mathrm{kV}-300 \mathrm{kV}$, because of the reduced exposure of the sample to the electron beam. The sensitivity of the BSE method on the nanoscale increases at medium to low voltages with a bulk sample. In addition, BSE imaging can also be simple and effective in the study of surfaceloaded nanocatalysts on bulk supports, including novel nanosupports employed in industrial reactions such as fuel cells and polymerization, compared to conventional TEM and STEM analyses which require electron-transparent samples. 
Fig.1a. BSE image of $\mathrm{Au} / \mathrm{C}$
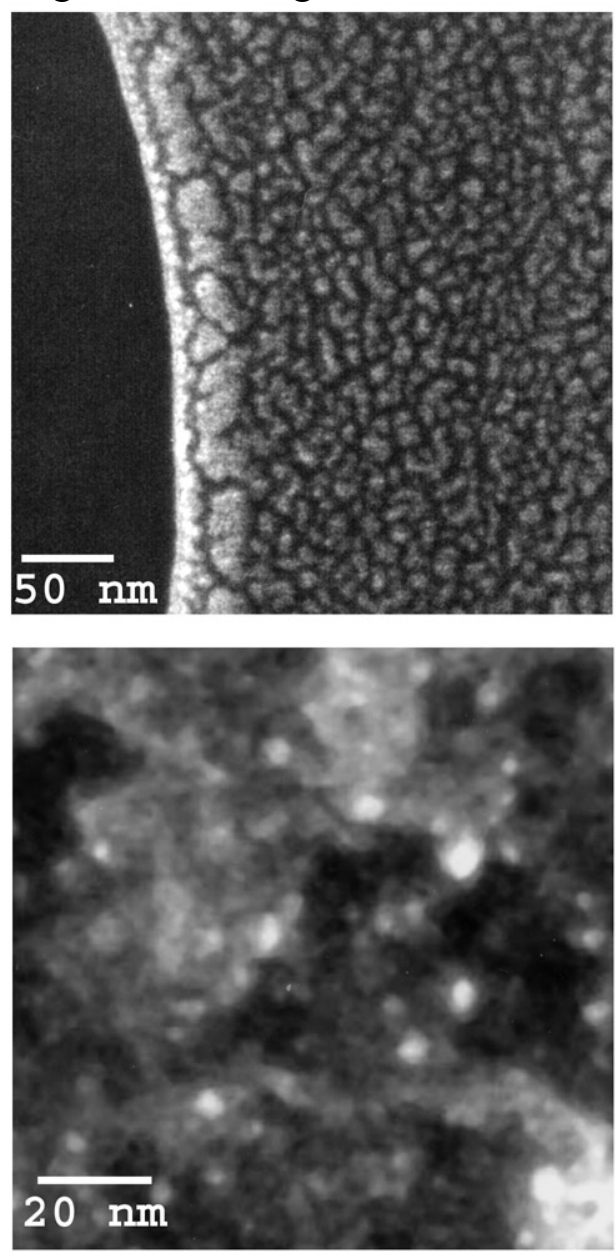

Fig.2a $\mathrm{Pd} / \mathrm{C}$ nanocatalyst using BSE
Fig.1(b). HAADF-STEM in FESEM
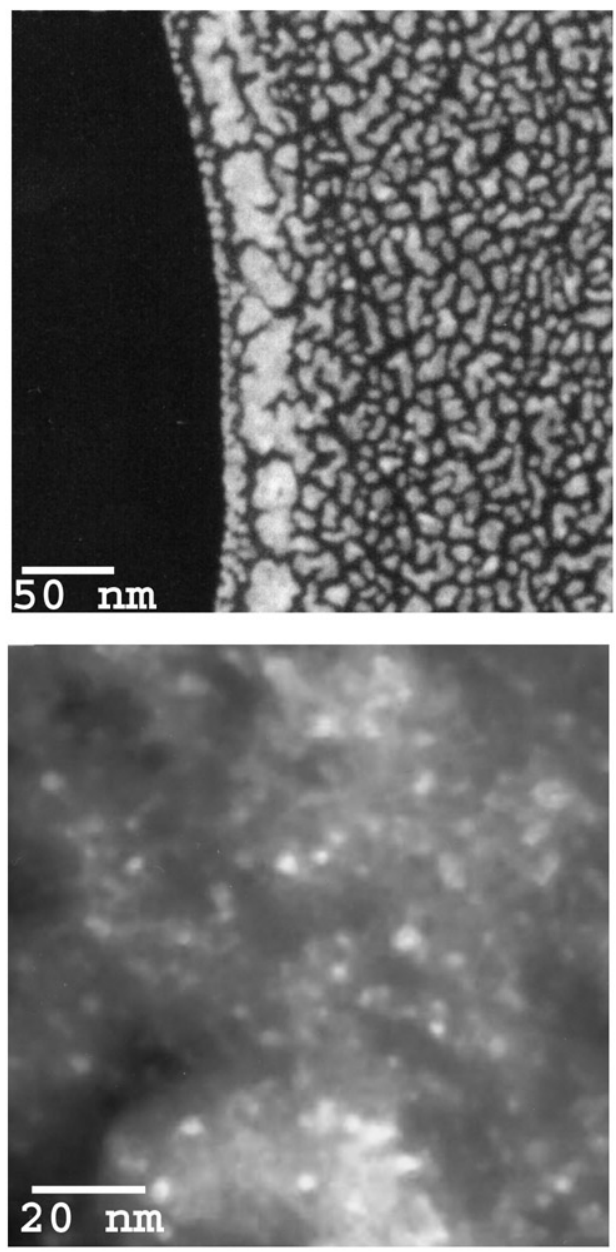

Fig. 2b. HAADF-STEM in FESEM

References

[1] E.D. Boyes, Mikrochim.Acta. 138 (2002) 225-234.

[2] P.A. Midgeley, M. Weyland, J.M. Thomas, P.L. Gai and E.D. Boyes, Angew.Chemie (International Edition). 41 (No.20) (2002) 3804-3807.

[3] P.L. Gai et al. Microsc. and Microanal. 6 (2000) 335- 342.

[4] P.L. Gai and E.D. Boyes, Electron Micr. in Het.Catalysis (IOPP, UK, USA) 2003.

[5] L.G. Hanna (CR\&D, DuPont) is thanked for technical assistance. 